\title{
Investigation of limbal touch sensitivity using a Cochet-Bonnet aesthesiometer
}

\author{
J G Lawrenson, G L Ruskell
}

\begin{abstract}
Following the observation of complex sensory receptors concentrated within the palisade zone of the human conjunctiva, this study sought to measure limbal touch sensitivity using a Cochet-Bonnet aesthesiometer. Touch sensitivity was found to be significantly higher in the palisade zone compared with the adjacent conjunctiva. A comparison between temporal and inferior limbus showed a greater median sensitivity for the temporal zone. There was a significant reduction in touch sensitivity with age, but not with iris colour or contact lens wear. These data, showing a higher touch sensitivity for the palisade zone, provide indirect evidence for a role of complex nerve endings in mechanoreception.

(Brf Ophthalmol 1993; 77: 339-343)
\end{abstract}

Current knowledge regarding the tactile sensitivity of the anterior eye segment is largely restricted to the sensitivity of the cornea, ${ }^{12}$ and less attention has been given to the conjunctiva. Using graded hairs of different calibres Von Frey and Strughold ${ }^{3}$ noted a considerable decline in sensitivity on crossing the limbus into the conjunctiva where large regional variations were apparent. Thresholds were generally lower in the region of the fornix and at the eyelid margin. Other studies ${ }^{45}$ also recorded a sensitivity profile across the conjunctiva showing marked regional variations with threshold values ranging from only $2 \times$ to more than $1400 \times$ greater than the central cornea. In contrast, Norn ${ }^{6}$ found a fairly uniform sensitivity across the conjunctiva, although he also found an enhanced sensitivity at the lid margin.

Our recent description ${ }^{7}$ of large numbers of complex (corpuscular) nerve endings in the

Applied Vision Research Centre, Department of Optometry and Visual Science, City University, London ECIV OHB

J G Lawrenson

G L Ruskell

Correspondence to: Dr J G Lawrenson, Reta Lila Weston Institute of Neurological Studies, University College and Middlesex School of Medicine, Riding House Street, London WIP 7PN

Accepted for publication 27 January 1993

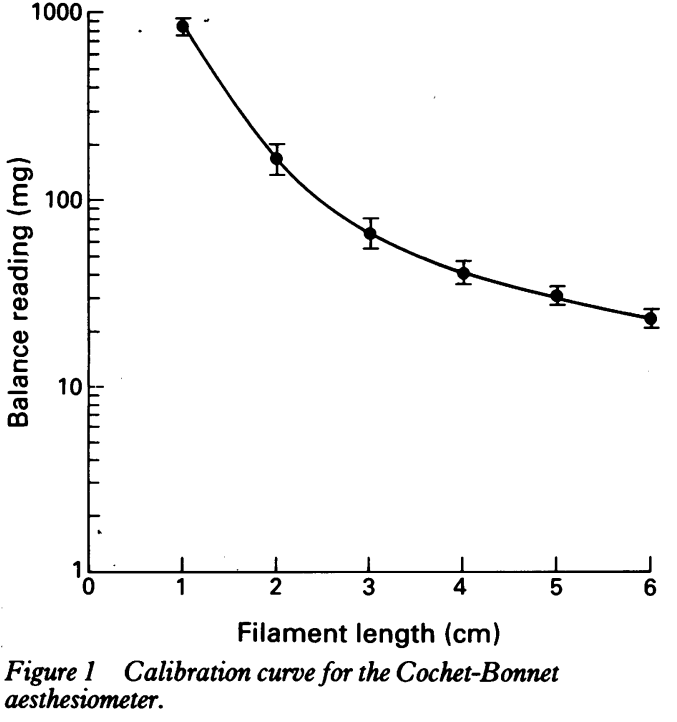

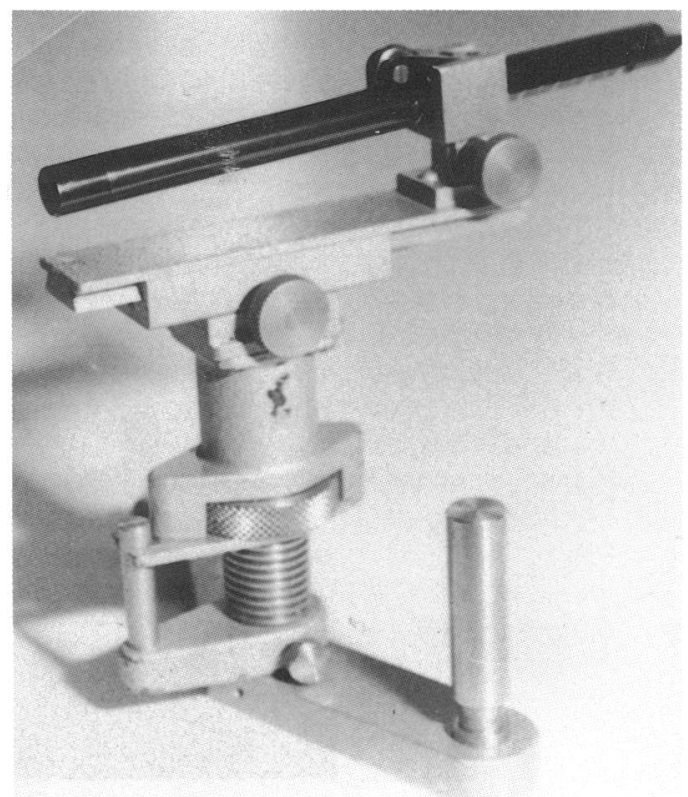

Figure 2 The aesthesiometer attached to an applicator which allows precise manipulation of the orientation of the filament.

limbal conjunctiva of the human eye led to the present study. These terminals were concentrated within a narrow annular zone of limbal conjunctiva coincident with the palisades of Vogt. ${ }^{8}$ Within this zone local and intersubject variations were found in both incidence and distribution of limbal corpuscles. ${ }^{9}$

This study represents our first efforts to determine a functional role for these putative receptors. Since morphological data favour mechanoreception, the touch sensitivity of the limbal conjunctiva was investigated using a Cochet-Bonnet aesthesiometer. The variables of age, iris pigmentation, and contact lens wear were also considered because of their known influence on corneal sensitivity. ${ }^{12}$

\section{Subjects and methods}

SUBJECTS

Subjects consisted of 28 non-contact lens wearers, 16 women and 12 men, with a mean age of 42 years (range 15-81 (SD 17.8) years). The sensitivity of 22 contact lens wearers was also determined. These were divided into rigid gas permeable (RGP) $(n=12)$, and hydrogel wearers $(n=10)$. RGP wearers comprised eight women and four men with a mean age of 34.3 years (range 21-59 (SD 10.6) years). Mean duration of lens wear was 10.0 years (range 0.5 20 (SD 5.3) years). Hydrogel wearers were all women with a mean age of 33 (range 18-59 (SD 11.4)) years. Mean duration of wear was $7 \cdot 5$ (range 0.5-13 (SD 4.0)) years. 


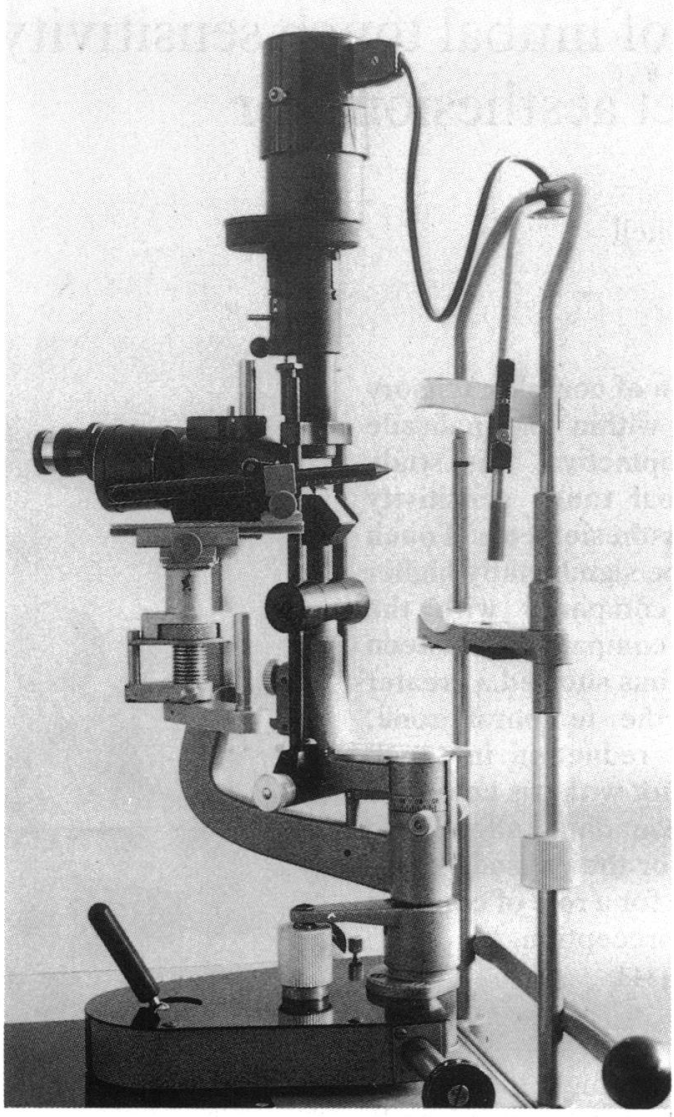

Figure 3 The apparatus attached to a slit-lamp microscope.

All contact lens wearers had worn their lenses on a daily basis for a minimum of 8 hours per day.

\section{Methods}

Touch thresholds were determined using a Cochet-Bonnet aesthesiometer with a $0.12 \mathrm{~mm}$ nylon monofilament. The instrument was calibrated by clamping it onto a laboratory stand and suspending it above the pan of a sensitive digital analytical balance (accurate to $1 \mathrm{mg}$ ). The filament, viewed through a binocular viewing system, was then smoothly lowered towards the pan. The criterion of first visible bending was used to standardise the applied pressure. The

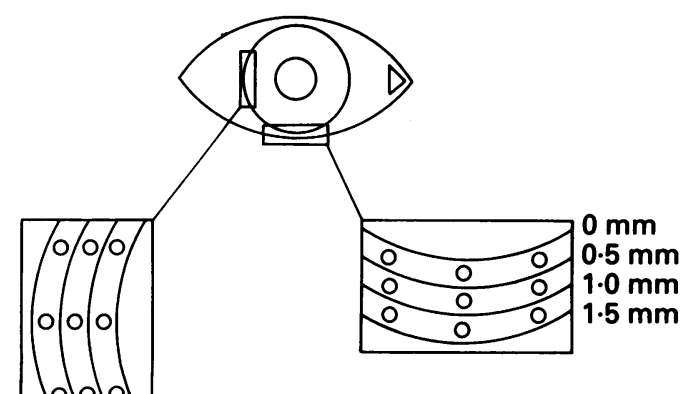

Zone $1=0-0.5 \mathrm{~mm}$

Zone $2=0.5-1.0 \mathrm{~mm}$

Zone $3=1.0-1.5 \mathrm{~mm}$

Figure 4 Stimulus test locations within three $0.5 \mathrm{~mm}$ wide concentric zones of limbal conjunctiva. A total of 18 points were divided equally between inferior and temporal limbus. mean of 20 balance readings was then plotted against filament length (Fig 1).

For measurement of touch thresholds the aesthesiometer was mounted on a slit-lamp microscope using a modified Bleshoy ${ }^{10}$ applicator (Figs 2 and 3). This apparatus allows fine control of filament application, viewed under magnification through the slit-lamp observation system. Thus, the nylon monofilament approached the conjunctival surface both smoothly and perpendicularly with accurate placement of the stimulus.

The subject was asked to indicate when the stimulus was felt. Longer lengths were utilised first. The criterion for threshold was the filament length which gave a $50 \%$ positive response from four stimulus applications. To test subject reliability a number of 'dummy runs' where the filament did not contact were included. Contact lens wearing subjects were instructed to wear their lenses for a minimum of $\mathbf{3}$ hours on the day of testing and thresholds were measured immediately on lens removal.

The touch threshold was determined at 18 locations within a $1.5 \mathrm{~mm}$ wide pericorneal area divisible into three $0.5 \mathrm{~mm}$ wide concentric zones beginning at the anterior termination of the palisades (Fig 4). Thus, test locations within zone 1 corresponded approximately to a midpalisade position. Stimuli were divided equally between zones and between temporal and inferior limbus.

Since the aim was to ensure an accurate repeat testing of the same conjunctival location, in order to minimise mechanical trauma it was decided to limit testing to three filament lengths $(4 \mathrm{~cm}, 3 \mathrm{~cm}$, and $2 \mathrm{~cm}$ ) giving threshold scores of $\geq 4 \mathrm{~cm}, \geq 3 \mathrm{~cm}, \geq 2 \mathrm{~cm}$, or $<2 \mathrm{~cm}$. To make these values more amenable to analysis they were converted to an arbitrary sensitivity scale (Table 1).

For the determination of iris colour the classification system of Seddon $e t a l^{11}$ was used.

\section{STATISTICAL ANALYSIS}

Because only three filament lengths were used, which were then converted onto an ordinal sensitivity scale, non-parametric statistical tests were utilised in the analysis ${ }^{12}$ and the determination of medians rather than means was appropriate.

For a single sample case paired measures were analysed using the Wilcoxon signed ranks test. Two independent samples were compared using the Mann-Whitney test. The Friedman two way analysis of variance (ANOVA) was used when more than two related samples were tested, and the Kruskal-Wallis one way ANOVA for more than two independent samples.

\section{Results}

INTERZONE VARIATION IN SENSITIVITY IN NONCONTACT LENS WEARERS

A comparison of the medians for all three zones (Fig 5) showed a fall off in sensitivity with increasing distance from the cornea. The differences in median sensitivity between zones 1 and 
Table 1 Sensitivity conversion scale

\begin{tabular}{ll}
\hline $\begin{array}{l}\text { Theshold } \\
\text { (cm) }\end{array}$ & $\begin{array}{l}\text { Sensitivity scaling } \\
\text { (arbitrary units) }\end{array}$ \\
\hline$<2$ & 1 \\
$\geq 2$ & 2 \\
$\geq 3$ & 3 \\
$\geq 4$ & 4 \\
\hline
\end{tabular}

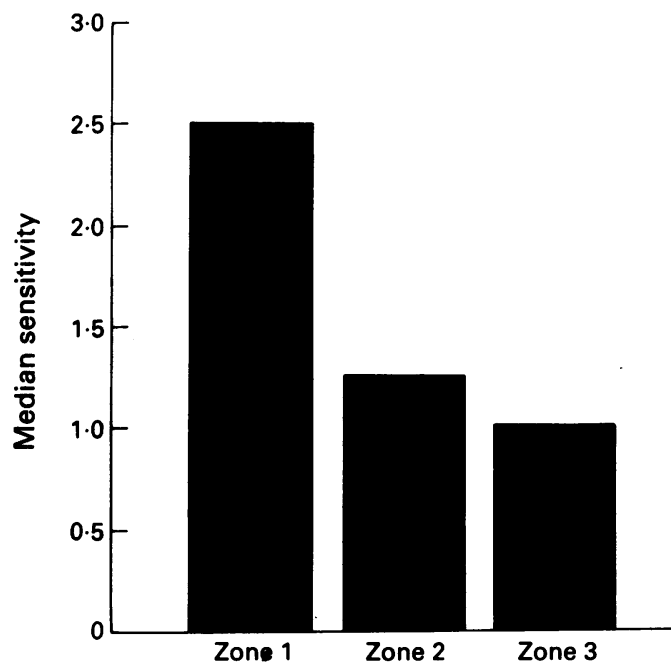

Figure 5 Variations in median sensitivity between zones 1, 2 , and 3 of non-lens wearers.

2 , and also between zones 1 and 3, were significant $(\mathrm{p}<0.05)$, whereas that between zones 2 and 3 was not $(p>0.05)$.

A greater sensitivity was found for the temporal (median 2) compared with the inferior limbus (median 1). This difference was significant $(p<0.05)$. Individual differences between medians for the temporal and inferior zones were plotted in order of increasing subject age (Fig 6). For subjects in the latter half of the group (range 40-81 years), there is a tendency for the difference between medians to be zero.

\section{EFFECTS OF CONTACT LENS WEAR}

RGP lens wearers $(n=12)$ and hydrogel wearers $(n=10)$ were analysed independently. The median sensitivity of individuals was calculated separately for zones 1,2 , and 3 and an overall group median was determined for each zone. These were plotted against the equivalent values for a group of age-matched non-contact lens wearers.

A plot of the median sensitivity for zones 1,2 , and 3 for RGP wearers versus age-matched controls (Fig 7) appeared to show a trend

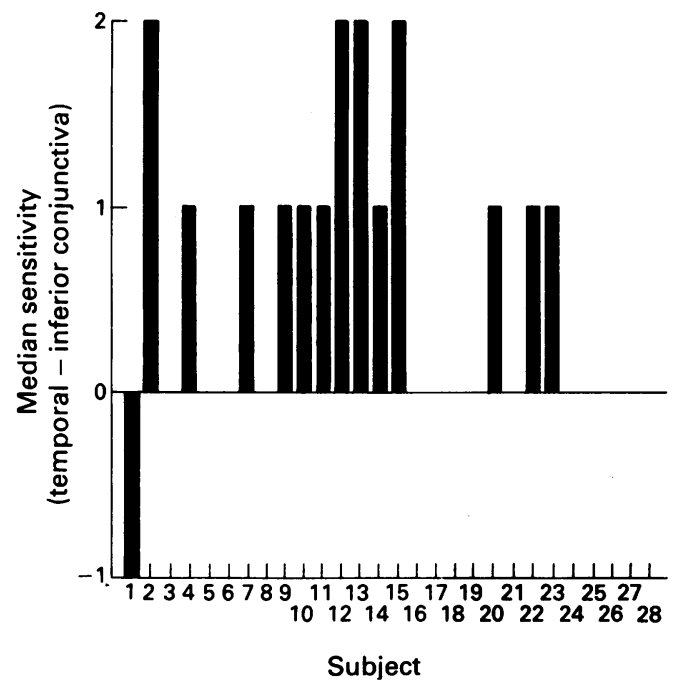

Figure 6 The difference in median sensitivity between temporal and inferior conjunctiva for each subject (nonwearers) plotted in order of increasing subject age.

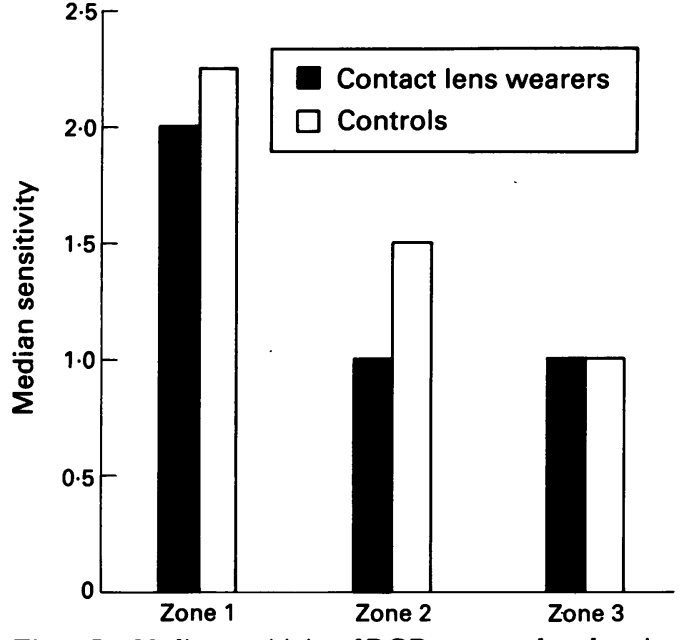

Figure 7 Median sensitivity of RGP wearers plotted against age matched non-wearers (mean age of lens wearers $34 \cdot 3$ $(S D 10 \cdot 6)$ years, mean age of controls 34.9 (SD 10.8) years).

towards a lower sensitivity in the contact lens wearers, but it was not found to be statistically significant $(\mathrm{p}>0.05)$. A similar trend was not observed in hydrogel wearers (Fig 8).

The influence of length of wear was investigated separately and not found to be significant $(\mathrm{p}>0.05)$

Because of the lack of a significant difference in sensitivity between contact lens wearers and non-lens wearers, it was felt valid to pool together all experimental groups for subsequent analyses.

\section{EFFECTS OF AGE}

The overall median sensitivity was plotted for all subjects $(n=50)$ against age (Fig 9$)$. The graph shows a large intersubject variability in limbal sensitivity, even within the same age decade. The overall trend however is a fall off in sensitivity with age.

The effect of age was analysed statistically by ranking subjects in order of increasing sensitivity. These assigned ranks were then grouped into four age profiles: $15-25,25-35,35-45$, and $>45$ years, and the ranks were then averaged

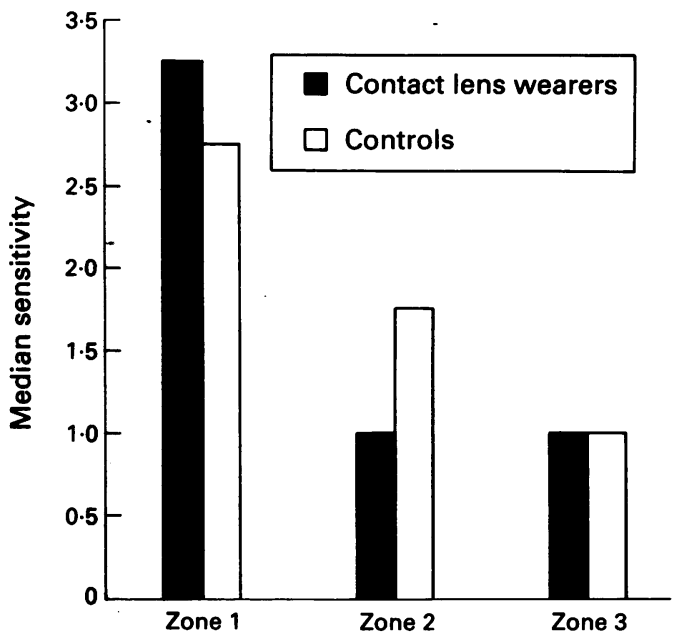

Figure 8 Median sensitivity of hydrogel wearers plotted against age matched non-wearers (mean age of lens wearers 33 $(S D 11 \cdot 3)$ years, mean age of controls $33 \cdot 8(S D 11 \cdot 0)$ years. 


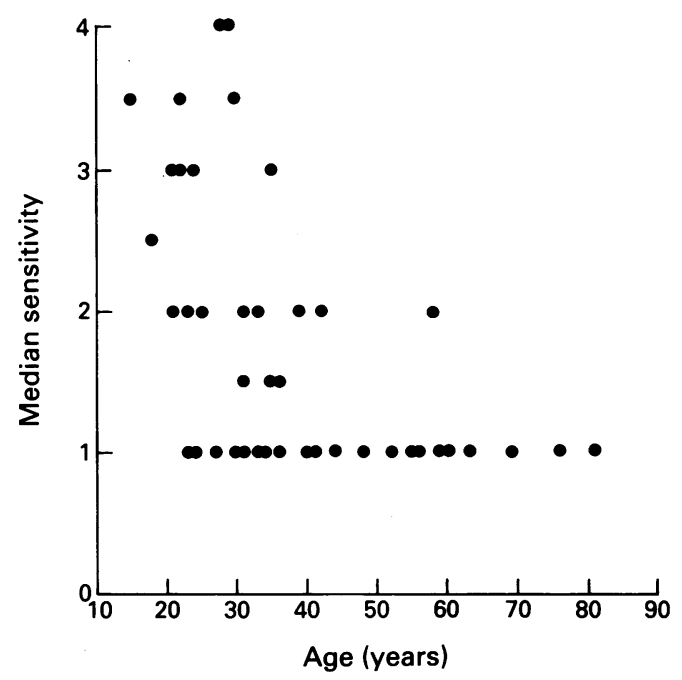

Figure 9 Graph showing overall sensitivity for all subjects plotted against age.

(Fig 10). The differences in sensitivity between the 15-25 group and the $35-45$ and $>45$ year groups were significant $(\mathrm{p}<0.01)$.

\section{EFFECTS OF IRIS COLOUR}

In order to compensate for any bias in the grouped data because of age, the subjects within each iris grouping were age matched. Subjects $(n=36)$ were ranked in order of increasing median sensitivity and then grouped according to their particular iris grading. The ranks were then averaged and these were then plotted against iris grade (Fig 11). No overall trend was apparent and these differences are not significant.

\section{Discussion}

A slit-lamp mounted aesthesiometer allows precise and repeatable stimulus application. With this apparatus it is possible to investigate systematically the touch sensitivity within a small conjunctival area. The present study has established a significantly higher sensitivity for the zone of the limbal palisades compared with the adjacent conjunctiva. Previous studies have shown a sharp decline in sensitivity immediately

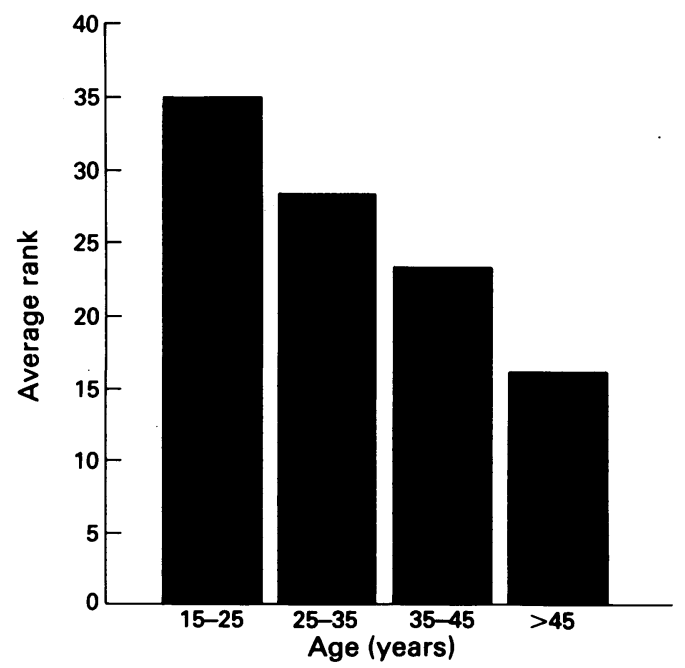

Figure 10 Effect of age on limbal sensitivity (ranked data).

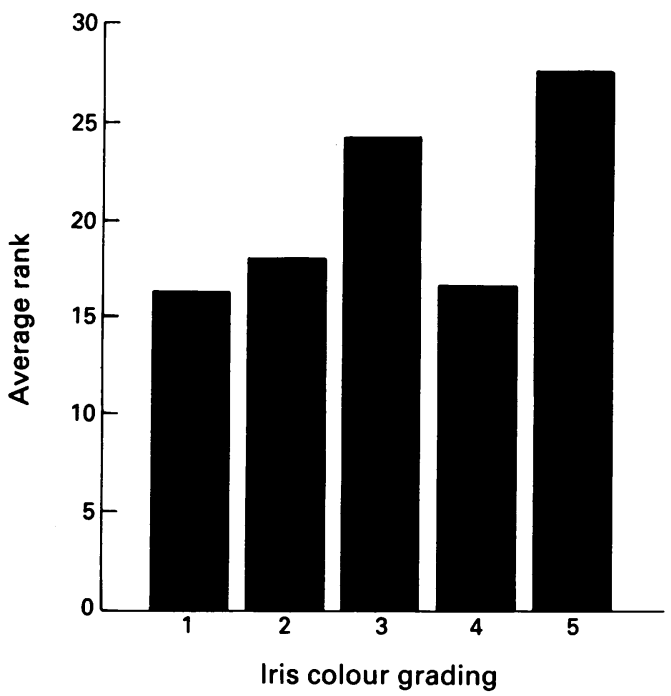

Figure 11 Graph showing the variation in median sensitivity with iris colour (ranked data). An iris is graded on a 1-5 scale in order of increasing pigmentation.

on crossing the corneoconjunctival junction. ${ }^{313}$ The limbal sensitivity values reported here, however, indicate that this fall off in sensitivity is less marked than previously documented.

It is worth examining these data in the light of differences in the pattern of sensory innervation of the cornea and conjunctiva. Many workers have shown that the cornea contains exclusively free (unspecialised) nerve endings which are numerous within the epithelium. ${ }^{1+}$ is The density of free nerve endings decreases markedly on passing into the conjunctiva where terminals within the epithelium are sparse.${ }^{16}$ However, in contrast to the cornea, the conjunctiva contains structurally specialised corpuscular nerve endings which are particularly numerous in the palisade zone. ${ }^{7}$ The finding of a significantly higher touch sensitivity in this region is consistent with a mechanoreceptive role for these nerve endings.

The proposed association of corpuscular nerve endings with touch sensitivity is strengthened by the observation of large individual variations in sensitivity, as a similar variation of receptor numbers was found. ${ }^{9}$ The present study showed that the temporal limbus had a greater median sensitivity than the inferior. Although quantitative histological data on limbal receptors ${ }^{9}$ indicate a non-uniform arrangement around the limbus, no distinct pattern has emerged from the limited number of eyes studied. However, since in $50 \%$ of subjects the median sensitivities of temporal and inferior zones were equal, many more eyes need to be examined histologically before any possible sectorial differences in receptor number become apparent.

The effects of contact lenses on corneal sensitivity are well known. Polymethylmethacrylate lenses cause a marked reduction in corneal touch thresholds $^{17}$ especially when worn for many years. ${ }^{18}$ The effect is less marked with hydrogel lenses, ${ }^{19}$ even when worn on an extended wear basis. The effects of contact lens wear on limbal conjunctival touch sensitivity was tested in this study for subjects wearing both rigid gas permeable and hydrogel lenses. However, no significant differences in limbal sensitivity were found 
for subjects wearing either lens type. This is not a surprising finding in view of the large intersubject variation in sensitivity which would most probably mask a small but possibly significant contact lens effect.

The decision to investigate iris pigmentation as a possible variable in limbal sensitivity was provided by the work of Millodot ${ }^{20}$ who showed that subjects with lighter coloured irides displayed a higher corneal sensitivity. It appears from the present study that such variation does not extend to the limbal conjunctiva.

In common with previous studies of cornea ${ }^{21}$ and bulbar conjunctiva ${ }^{45}$ the sensitivity of the limbus showed a significant reduction with age. Although there was a progressive fall off in sensitivity among adults, the greatest reduction was during the fourth and fifth decades.

We are grateful to Roger Buckley, director of the Contact Lens and Prosthetics Department, Moorfields Eye Hospital for allowing the use of the facilities of his department. The work was supported by a scholarship from the British College of Optometrists.

1 Boberg-Ans J. Experience in clinical examination of corneal sensitivity. Brf Ophthalmol 1955; 39: 705-26.

2 Millodot $M$. A review of research on the sensitivity of the cornea. Ophthalmol Physiol Opt 1984; 4: 305-18.

3 Frey $M$ von, Strughold $H$. Weitere Untersuchunden über das Verhalten von Hornhaut und Bindehaut des menschlichen Verhalten von Hornhaut und Bindehaut des menschlich
Auges gegen Beruhrüngesreize. Z Biol 1926; 84: 321-34.
4 Draeger J. Corneal sensitivity: measurement and clinical importance. Vienna: Springer Verlag, 1984.

5 Winter R, Draeger J, Rumberger E, Lucas L. Zur Sensibilitätsbestimmung an der Bindehaut- Methodik und erste klinische Befunde. Fortschr Ophthalmol 1986; 83: 176-8.

6 Norn MS. Conjunctival sensitivity in normal eyes. Acta Ophthalmol 1973; 51: 58-66.

7 Lawrenson JG, Ruskell GL. The structure of corpuscular

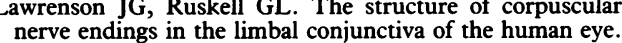
nerve endings in the limbat

8 Vogt A. Atlas of slit-lamp microscopy of the living eye. Berlin: Springer, 1921

9 Lawrenson JG. Corpuscular nerve endings in the human conjunctiva. PhD Thesis. London: City University, 1991.

10 Bleshoy $\mathrm{H}$. Corneal sensitivity in keratoconus. Trans $\mathrm{Br}$ Contact Lens Ass Conf 1986: 9-12.

11 Sedden JM, Sahagian CR, Glynn RJ, Sperduto RD, Gragoudas ES. Evaluation of an iris color classification system. Invest Ophthalmol Vis Sci 1991; 31: 1592-8.

12 Seigel S. Non-parametric statistics for the behavioral sciences. New York: McGraw-Hill, 1956.

13 Draeger J. Modern aesthesiometry. Contribution to corneal metabolism after anterior segment surgery. Trans metabolism after anterior segment
Ophthalmol S oc UK 1979; 99: 247-51.

$14 \mathrm{Lim}$ HK, Ruskell GL. Corneal nerve access in monkeys. Graefes Arch Clin Exp Ophthalmol 1978; 208: 15-23.

15 Schimmelpfennig B. Nerve structure in the human central corneal epithelium. Graefes Arch Clin Exp Ophthalmol 1982; 218: $14-20$.

16 Ruskell GL. Innervation of the conjunctiva. Trans Ophthalmol Soc UK 1985; 104: 390-5.

17 Millodot $M$. Effect of hard contact lenses on corneal sensitivity and thickness. Acta Ophthalmol 1975; 53: 576-84.

18 Millodot M. Effect of long term wear of hard contact lenses on corneal sensitivity. Arch Ophthalmol 1978; 96: 1225-7.

19 Millodot M. Effect of soft lenses on corneal sensitivity. Acta Ophthalmol 1974; 52: 603-8.

20 Millodot $M$. Do blue eyed people have more sensitive corneas than brown eyed people? Nature 1975; 255: 151.

21 Millodot $M$. The influence of age on the sensitivity of the Millodot M. The influence of age on the sensitivity
cornea. Invest Ophthalmol Vis Sci 1977; 16: 240-2. 